\title{
Clinical Diagnostics, Therapy and Outcome After Basilar Artery Thrombosis
}

\author{
Anja Lohse ${ }^{*}, 1$, Christoph Preul ${ }^{1}$, Otto W. Witte ${ }^{1}$ and Stefan Isenmann ${ }^{1,2}$ \\ ${ }^{1}$ Hans Berger Clinic for Neurology, Jena University Hospital, Friedrich-Schiller-University, Erlanger Allee 101, D- \\ 07747 Jena, Germany \\ ${ }^{2}$ Department of Neurology, Helios Clinic Wuppertal, Heusnerstr. 40, D-42283 Wuppertal, and University of \\ Witten/Herdecke, Germany
}

\begin{abstract}
Acute basilar artery occlusion is a relatively rare but life threatening condition requiring rapid diagnosis and treatment. The circumstances and predictive value for outcome in these patients are not well documented. 88 patients with basilar artery occlusion treated in the Department of Neurology at Jena University Hospital from 1998 to 2007 were identified and studied. Of these, 45 were males and 43 females, aged between 20 and 92 years (median, 63 years). In $60.2 \%$, MRI with MR angiography led to the diagnosis; $13.6 \%$ of all cases were diagnosed by cranial CT. 45 patients were treated with thrombolytic therapy with rt-PA (44/45) or urokinase (1/45; in 1999). Out of 45 patients, eight (17.7\%) initially received systemic (i.v.) thrombolytic treatment.

Recent research has suggested that for patients with unambiguous basilar artery thrombosis, the only promising treatment option is early thrombolytic treatment. The major clinical goal is to enable patients to live an independent life after the acute phase and, if required, rehabilitation. Consequently, this challenges the concept of a "thrombolysis at all costs" approach, especially in case of multi-morbidity, unidentifiable time frames, and comatose patients with regards to possible poor functional prognosis. An adequate therapy in the sense of an adaptable scheme, starting with immediate maximum therapy following diagnosis, and with defined decision points for possible therapeutic limitations at later stages of the disease course after ensuring poor prognosis may require further testing, depending on clinical conditions and the patients' documented, or presumable wishes.
\end{abstract}

Keywords: Basilar artery thrombosis, basilar artery occlusion, thrombolysis, stroke.

\section{INTRODUCTION}

Thrombosis of the basilar artery is a relatively rare yet serious type of ischaemic stroke that causes death in up to $95 \%$ of all cases, unless quick and sufficient therapy is provided. Basilar artery thrombosis may be caused by local thrombosis on the basis of a prominent stenosis of the basilar artery, arterio-arterial embolism with an upstream stenosis, or, in even rarer cases, dissection of the vertebral artery, or cardiac/ aortic (arterio-arterial) embolism [39].

Definite diagnosis can be reached using different imaging methods, such as computed tomography (CT) and magnetic resonance imaging (MRI), or by conventional cerebral angiography. In addition, ischemia in brain regions which are supported by the basilar artery (i.e. brainstem, cerebellum, thalami, and the territory of the posterior cerebral arteries) can be differentiated from bleedings and other lesion types, e.g. tumors or inflammatory lesions.

Up to the 1980 s, therapeutic options with basilar thromboses were very limited, and the course of the disease was mostly lethal, especially since elaborated intensive medical care of stroke patients had not yet been established. Thus, the mortality of patients suffering from

*Address correspondence to this author at the Hans Berger Clinic for Neurology, Jena University Hospital, Friedrich-Schiller-University Jena, Erlanger Allee 101, D-07747 Jena, Germany; Tel: ++49 +3641 9323421; Fax: ++49+3641 9323422; E-mail: Anja.Lohse@med.uni-jena.de angiographically identified basilar thrombosis was between 80 and $90 \%$ [1-3]. The introduction of intra-arterial (i.a.) use of fibrinolytics in the early 1980s led to a significant improvement of prognosis. However, even after successful recanalisation of the basilar artery, mortality still reached an average of $50 \%$ [4-6]. This may have been due to the fact that vulnerable brain tissue in critical brain stem structures has been irreversibly damaged before initiation of sufficient therapy and vessel recanalisation.

Nowadays, the prognosis of basilar artery thrombosis is still poor. Thus, it is common sense that nearly any promising intervention appears justified [7], because of the otherwise disastrous outcome. Due to the rapid progress in endovascular methods within the past 25 years, especially local intra-arterial fibrinolysis [8], angioplasty [9], and more recent development in fibrinolytics, more refined therapies of brain stem strokes have been established and systematically analyzed [19].

Today, most clinical neurologists would agree that thrombolytic therapy is an efficient strategy for acute basilar artery occlusion. This therapy can help save lives as well as prevent or mitigate neurological symptoms and physical handicaps. Complications of this therapy can be predicted on a general, but not on an individual basis. Occasionally, this treatment may cause more harm than good to the patient [10]. Unfortunately, there is little data available on functional outcome as well as survival rates in follow-up examinations [11]. In order to contribute to these issues, 
accessible data of 88 consecutive patients with basilar artery thrombosis from a single center have been studied. All patients have received medical treatment in the Department of Neurology at Jena University Hospital from 1998 to 2007 - i. e., for a decade.

\section{MATERIAL AND METHODS}

88 consecutive patients suffering thrombosis of the basilar artery treated in the Department of Neurology at Jena University Hospital from 1998 to 2007 were retrospectively analyzed. All patients had either been diagnosed with basilar artery occlusion, or presented with stoke patterns typical of basilar artery thrombosis on cerebral imaging (CT or MRI). Of these, 45 were males and 43 females, aged between 20 and 92 years (median, 63 years).

Using available files, data were compiled about the medical history, diagnoses, etiology, risk factors, therapy, and outcome (using mRS) of these patients. Data were systematically collected and statistically analyzed using SPSS for Windows 11.5 (SPSS Inc., Chicago, USA; normally distributed, unpaired simple T-Test).

\section{RESULTS}

Among the 88 patients, there were 43 women (48.9\%) and 45 men $(51.1 \%)$. The average age was $64.1 \pm 14.8$ years (20-92 years). In our study male patients were on average twelve years younger than female patients $(p<0.05)$. All patients had been diagnosed with ischemic stroke in the basilar artery territory through cerebral imaging. In $60.2 \%$, MRI with MR angiography led to the diagnosis; only $13.6 \%$ of all cases were diagnosed by cranial CT.

The affected brain areas were: cerebellum, pons, thalamus, midbrain, and posterior cerebral artery territory. Some patients only had a minor stroke in the posterior cerebral artery territory, others had large areas affected, partly on both sides (Table 1, Fig. 1). Accordingly, the initial symptoms were rather heterogeneous (Fig. 2). Most common were impaired level of consciousness $(71 / 88)$, cranial nerve palsy (58/88), limb paresis (50/88), dysarthria (40/88), dizziness/vertigo (34/88), nausea and vomiting (25/88), respiratory failure $(21 / 88)$, headache $(10 / 88)$, and sensory deficits $(9 / 88)$.

Table 1. Frequency of Stroke Areas

\begin{tabular}{|c|c|c|c|}
\hline S. No & Stroke Localization & $\mathbf{n}$ & $\%$ \\
\hline \hline 1 & Pontine Ischemia & 63 & 71,6 \\
\hline 2 & Cerebella Ischemia & 62 & 70.5 \\
\hline 3 & Thalamic Ischemia & 45 & 51,1 \\
\hline 4 & Midbrain Ischemia & 43 & 48,9 \\
\hline 5 & Posterior artery territory Ischemia & 31 & 35,2 \\
\hline
\end{tabular}

$\mathrm{n}=$ number of patients (of 88) with stroke distribution.

In 45 out of 88 cases $(51.1 \%)$, symptoms evolved gradually or even fluctuating. Mild symptoms had been present for up to two months in individual cases before the acute event qualifying for admission. These symptoms have been retrospectively classified as symptoms of basilar artery pathology. In other patients, symptoms occurred as transitory ischemic attacks (TIAs) with complete remission within a few minutes before admission and cerebral imaging. In a large group of patients $(50 / 88)$ symptoms such as impaired consciousness occurred suddenly and unexpectedly, often with evolution of coma within few hours.

None of these patients had been definitely diagnosed with a congenital clotting disorder or propensity to thromboses before the actual occurrence of basilar artery thrombosis. 24 patients $(27.3 \%)$ had had myocardial infarction, coronary heart disease, deep vein thrombosis, pulmonary embolism, or persistent foramen ovale (PFO)
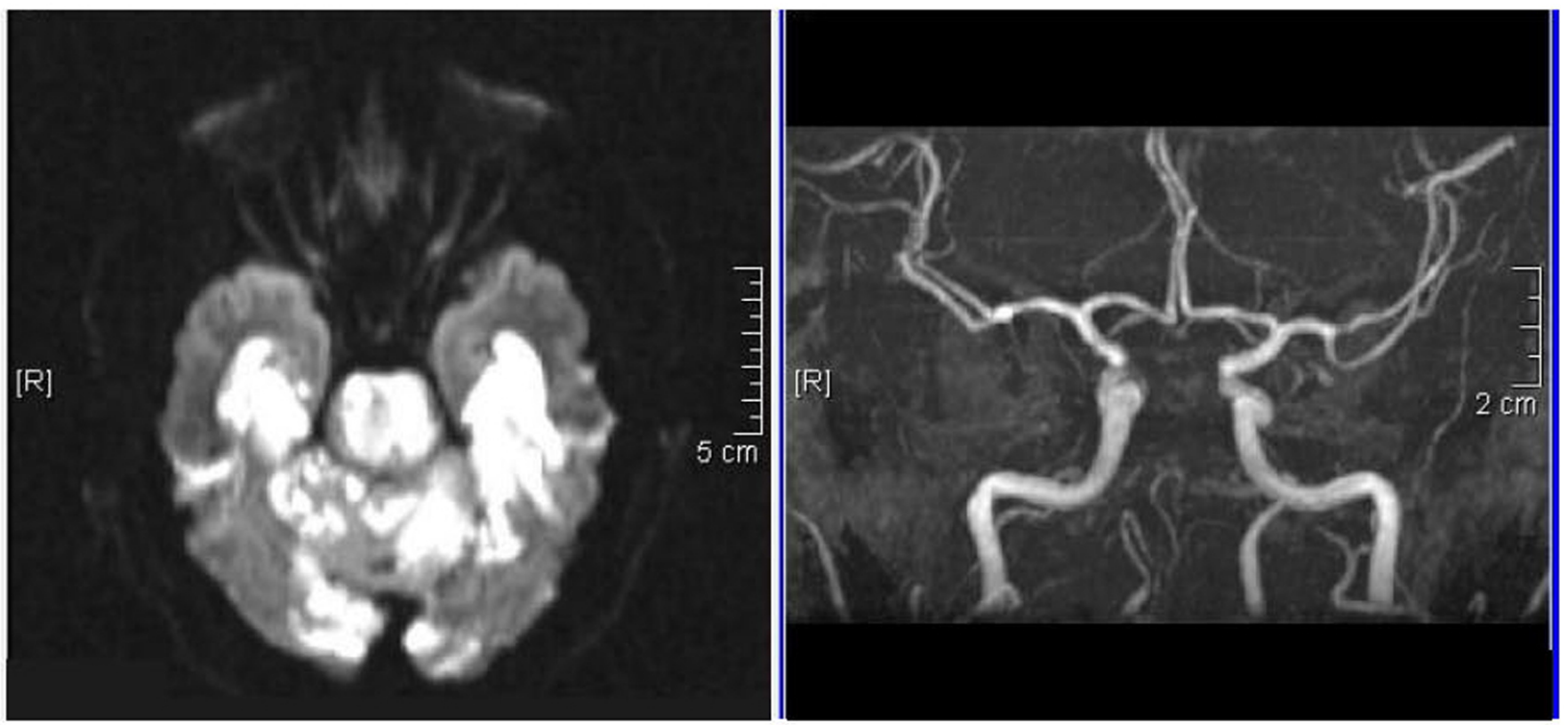

Fig. (1). MRT four days after patients' admission to the hospital. DWI shows bilateral (complete) pontine, cerebellar, and bilateral infarction of the posterior circulation, respectively. In the TOF angiography basilar artery is missing. 


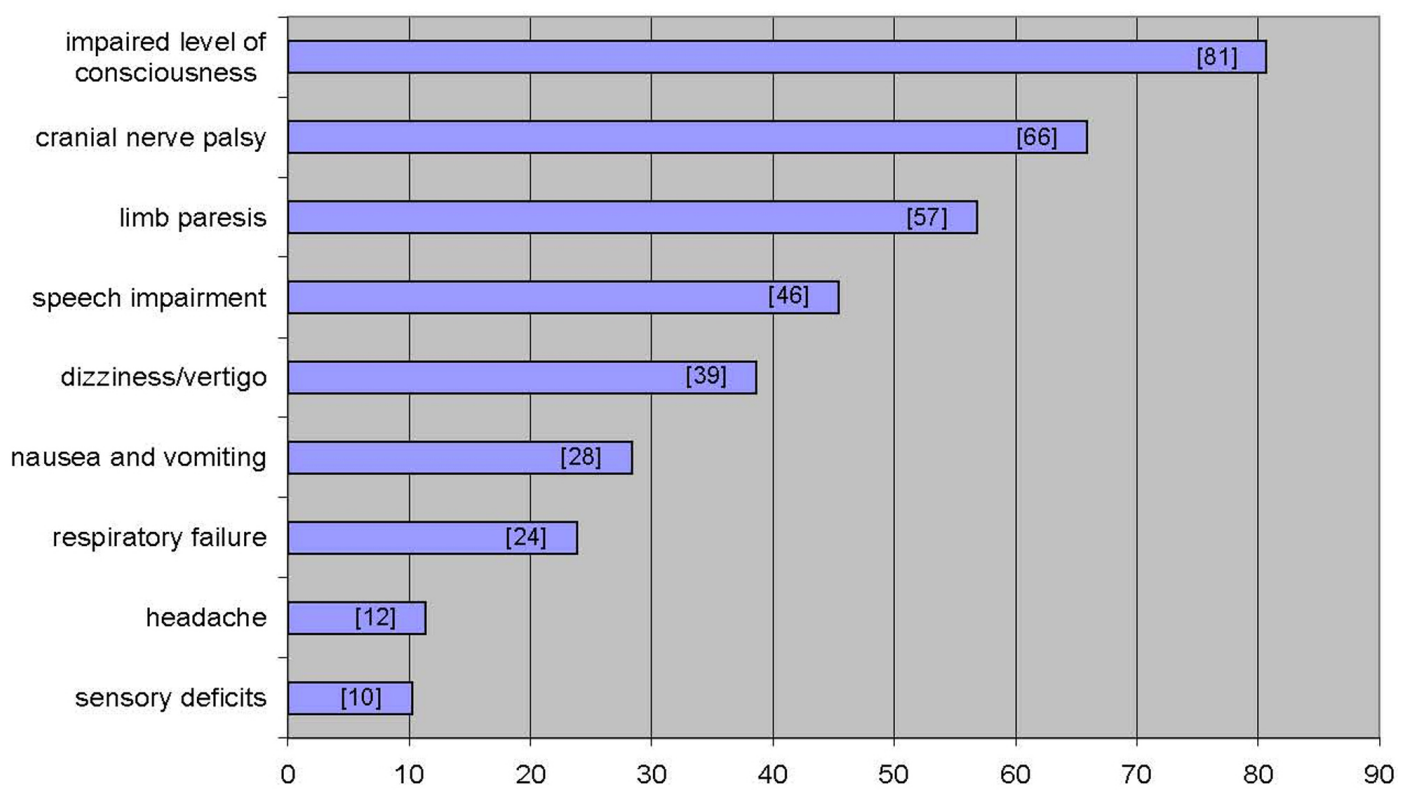

Fig. (2). Initial symptoms of basilar artery thrombosis (percentages).

previously. However, no former basilar artery pathology was present in any of the patients. Based on imaging criteria, local thrombosis of the basilar artery was present in 41 out of 88 patients $(53.4 \%)$, while in 47 out of 88 patients $(46.6 \%)$ the symptoms were attributed to embolism. This differentiation is based on the angiographic findings and the appearance of the basilar artery after recanalisation: 45 patients were treated with thrombolytic therapy with rt-PA $(44 / 45)$ or urokinase (1/45; in 1999). Out of 45 patients, eight $(17.7 \%)$ initially received systemic (i.v.) thrombolytic treatment. These patients had either been referred to the University Hospital Jena from peripheral hospitals and i.v. thrombolysis has been initiated as 'bridging therapy' to bridge the time delay to arrival at the center. Three of these eight patients (37.5\%) also received local (i.a.) thrombolytic therapy subsequently.

Two patients failed to receive local thrombolytic therapy owing to technical inaccessibility of both vertebral arteries. In one patient, the basilar artery remained occluded despite a maximum rt-PA dose $(100 \mathrm{mg})$ so that mechanical recanalisation through a catheter was attempted and achieved.

26 out of 45 patients $(57.8 \%)$ who had received thrombolytic therapy showed clear clinical improvement (using mRS) and sufficient collateralization. Therefore, in these cases, there was no need for further treatment. Additionally, another patients basilar artery remained occluded in spite of systemic thrombolytic therapy with urokinase. This patient died later from sepsis with multi organ failure. Three out of 45 patients $(6.8 \%)$ were also treated with a GP-IIb/IIIa inhibitor. Two of them had complete recanalisation. Ensuing therapeutic anticoagulation with i.v. heparin was completed in 39 out of 45 patients (86.7\%).

43 out of 88 patients $(48.9 \%)$ have not been treated with thrombolytic therapy. This was due to uncertain time frame (with already demarcating posterior circulation infarction), or with time frames covering ten hours or more (14/43;
$32.6 \%$ ), a generally reduced patient condition (already before the actual event), or multi-morbid patients $(8 / 43 ; 18.6 \%)$, significant stroke demarcation on cCT or MRI (16/43; $37.2 \%$ ), and spontaneous recanalisation (19/43; 44.2\%). This list allowed for more than just one cause per patient.

Patients were tested for common risk factors of arteriosclerosis as well as cardiac diseases. Table $\mathbf{2}$ illustrates the major risk factors. Four male patients did not reveal any of the known risk factors tested; eight patients revealed five risk factors, whereas two patients (both female) had even six risk factors.

Out of 88 patients, 66 had to be fed through a nasogastric tube for at least 24 hours to ensure sufficient supply with medication and nutrition, owing to impaired swallowing or impaired vigilance. 30 patients (34\%) required percutaneous endoscopic gastrostomy treatment for long-term swallowing impairment.

69 out of 88 patients (78.4\%) faced complications during their hospital stay, most commonly pneumonia with 54 out of 88 patients $(61.4 \%)$. 38 out of these 54 patients $(70 \%)$ had to be mechanically ventilated. The reasons for intubation were either protective intubation to avoid aspiration, impaired consciousness, cardiopulmonary reanimation, sedation for imaging diagnostics, or respiratory insufficiency. Arterial hypotension requiring volume therapy or even transient catecholamines occurred in $35.2 \%$ of all cases (31/88 patients). Bleeding complications such as gastro-intestinal bleedings and cerebral hemorrhage occurred in eleven patients (12.5\%), eight of whom (72.7\%) had received thrombolytic treatment.

The Modified Rankin Scale (mRS) was calculated from the reports (Fig. 3). 21 patients (23.8\%) had no or only few handicaps $(\mathrm{mRS} \leq 2)$ caused by the previously diagnosed basilar artery thrombosis and were not essentially handicapped in their everyday activities. 30 out of 88 patients $(34.1 \%)$ were moderately or strongly handicapped, sometimes even to an extent that required support in their everyday activities (mRS 3-4). Twelve of these patients 
Table 2. Sex-linked Distribution of Risk Factors and their Frequency; Most Risk Factors were First -Order Factors

\begin{tabular}{|c|c|c|c|c|c|c|}
\hline \multirow{2}{*}{ Risk Factor } & \multicolumn{3}{|c|}{ Number Of Patients (Absolute) } & \multicolumn{3}{|c|}{$\%$} \\
\hline & Total & Men & Woman & Total & Men & Woman \\
\hline Arterial Hypertension & 65 & 29 & 36 & 74 & 64 & 84 \\
\hline Age $>65$ Years & 54 & 20 & 34 & 61 & 44 & 79 \\
\hline Arial Fibrillation & 38 & 13 & 25 & 43 & 29 & 58 \\
\hline Hypercholesterolemia & 36 & 21 & 17 & 41 & 47 & 40 \\
\hline Diabetes Mellitus & 29 & 7 & 2 & 33 & 16 & 51 \\
\hline Nicotine Abuse & 17 & 12 & 5 & 19 & 27 & 12 \\
\hline Previous Stroke & 17 & 8 & 9 & 19 & 18 & 21 \\
\hline Alcohol Abuse & 11 & 9 & 2 & 13 & 20 & 5 \\
\hline
\end{tabular}

were referred as in-patients with massive functional handicaps $(\mathrm{mRS}=5)$ to rehabilitation. 25 patients $(28.4 \%)$ died in hospital $(\mathrm{mRS}=6)$.

Out of 21 patients with a mRS ranging from 0 to 2,15 $(71.4 \%)$ had been treated with thrombolytic therapy; only six patients $(28.6 \%)$ were in clinically good condition without thrombolytic therapy, but none of these patients without thrombolytic therapy had a mRS of 0 . Nine out of 30 patients $(30 \%)$ with a mRS of 3 to 4 had been treated with thrombolytic therapy, the other 21 patients $(70 \%)$ in this group did not have this therapy. Apart from this statistical analysis based on the mRS, we observed that out of all patients who had received thrombolytic therapy, twelve (27\%) died, whereas out of all patients without thrombolytic treatment, $13(30 \%)$ died. This observation can only be made if you form a group out of those nine patients who had been severely handicapped with a mRS of 5 together with three such patients $(25 \%)$ without thrombolytic therapy.

All patients were referred to the neurological intensive care unit, irrespective of thrombolytic treatment.

54 out of 86 patients $(61 \%$ of the total number, $86 \%$ of those who survived) were referred to rehabilitation. The duration of rehabilitation treatment varied from three days (rehabilitation cancelled by the patient) up to 162 days, the average being 65.3 days (median: 57 days). After the critical stage, four patients were referred back to local hospitals; no data are available on their further course.

25 out of 54 patients $(46.3 \%)$ were discharged home after rehabilitation. 16 out of these patients had been in the thrombolytic-therapy group. Another ten patients - five out of each group - were referred to community care or nursing homes. Two patients without thrombolytic therapy died during rehabilitation. For 17 patients, no reports on their rehabilitation or further progress were available.

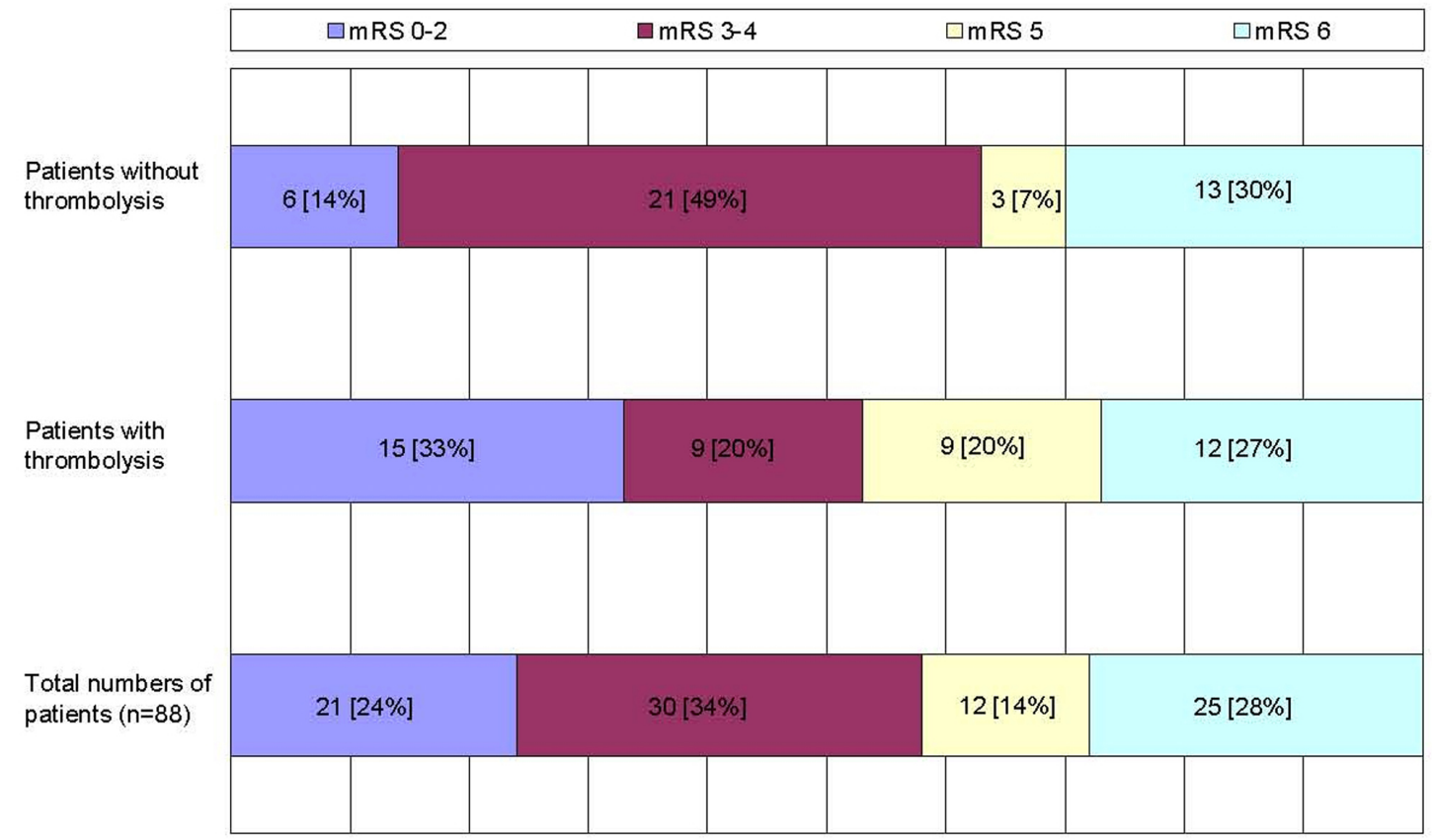

Fig. (3). Outcome of patients with basilar artery thrombosis modified Rankin scale (mRs) according to therapy (percentages in brackets). Mps: $0=$ no symptoms at all, $1=$ no significant disability despite symptoms, $2=$ slight disability, $3=$ moderate disability, $4=$ moderately severe disability, $5=$ severe disability, $6=$ death. 


\section{DISCUSSION}

The natural course of basilar artery is often lethal, and even survivors bear a high risk for mortality and morbidity [12,13]. The 'best' and most sufficient treatment methods are still a matter of debate. Since the 1980s, various case reports and follow-up studies on the outcome of acute vertebro-basilar thrombosis have been published $[2,4,5,8,14]$. The frequency of recanalisation after thrombolytic therapy has been estimated to be between 40 and $100 \%$, and the survival rate between 25 and $70 \%$, depending on patients selection and the type of therapy $[2,4,5,8,14-16]$. Unfortunately, the number of patients available was often too low to draw valid conclusions.

This retrospective study examines a relatively high number of patients from a single university center treated over a 10 years period of time. We set out to identify possible trends and independent factors influencing the rate of recanalisation and patients' clinical outcome.

In the Department of Neurology at the Friedrich Schiller University Hospital, Jena, 88 patients were identified as having basilar artery thrombosis between 1998 and 2007. This represents $1.7 \%$ of all strokes during that time in this single university center. There is no evidence of seasonal variation. Within the first three years (1998-2000), the number of patients with basilar artery thrombosis cases increased from five to 16 patients per year. This apparent increase may either represent realistic conditions or reflect the progress in alertness and diagnostic measures. In the ensuing years, the incidence was 8.8 patients per year with only minor variability.

As for the distribution of sexes, other authors' findings vary widely: Wijdicks et al. [17], identified 25 patients (11 females and 14 males), Sliwka et al. [18] identified 36 patients (8 females and 28 males), and Arnold et al. [19] identified 40 patients (18 females and 22 males). Our group consisted of 43 female and 45 male patients. Other studies may be more heterogeneous due to lower patient numbers or because of varying inclusion and exclusion criteria.

Regarding the patients' age, the literature gives an average of 60 years $[17,18,19]$, which is in agreement with an average age of 64 years in our patient cohort. In our study male patients were on average twelve years younger than female patients $(p<0.05)$. In contrast, the proportion of females was higher than that of males for nearly all risk factors (hypertension, diabetes, cardiac arrhythmia, previous stroke). This can be correlated with data from the literature concerning the incidence of cardiovascular risk factors. Women aged 60 or older suffer more often from type-two diabetes and arterial hypertension than men $[20,21]$. Atrial fibrillation, however, is generally more frequent in men [22]. In our study though, females were affected more often. Based on studies on arterial hypertension, $70 \%$ of all stroke patients are hypertensive $[23,24]$. Here, $74 \%$ of the patients had been diagnosed with arterial hypertension.

The clinical pictures of basilar artery thromboses were heterogeneous and typically included impaired consciousness, cranial nerve palsy, limb paresis as well as dysarthria (in descending order). Establishing an initial diagnosis, in connection with a clinically rather inhomogeneous condition, is difficult. In half of the patients the onset of the symptoms was sudden and acute, while in the other half of cases, symptoms progressed slowly and were fluctuating. Considering the most likely consequences, especially missing or delayed diagnosis, basilar artery thrombosis has to be generously included into differential diagnosis at an early stage.

In 1996, Wijdicks et al. reported an MRI rate of $12 \%$ for a reliable diagnosis; the CT rate was $44 \%$ [17]. $29.5 \%$ of the patients analyzed in this study were primarily examined using MRI including MR angiography, which detected basilar artery thrombosis or vessel recanalisation. $59.1 \%$ of these patients were initially sent to cranial CT and CT angiography with only $13.6 \%$ being identified with a pathology in the basilar artery territory. In contrast, in $60.2 \%$ of all patients MRI led to a reliable and immediate diagnosis. In conclusion, the clinical suspicion of basilar artery thrombosis should lead to early MRI and MR angiography in order to initialize respective treatment as soon as possible [36].

Reliable assessment of the brainstem with $\mathrm{CT}$ is difficult [25]. The essential indication for immediate cerebral CT is exclusion of cerebral hemorrhage as a prerequisite for initiation of systemic thrombolytic treatment before referral to a center with MRI facility, angiography, and a specialized stroke unit [35]. This was the case in 8 out of 45 patients who were treated by local thrombolysis after arrival at our center.

In our study, the proportion of patients receiving conventional angiography (DSA) was 68.2\%. However, because of its side effects, availability and pragmatic effort, DSA as a primary diagnostic procedure is only promising for patients with unambiguous symptoms clearly pointing to basilar artery thrombosis as was the case in only $3.4 \%$ of our patients [38].

In our study, cerebral ischemia caused by basilar artery thrombosis was located in pons, cerebellum, thalamus, midbrain, and posterior cerebral artery territories. The severity of clinical symptoms did not necessarily correlate with the extent of the ischemic area. By analyzing the extent of DWI lesions of four strongly affected patients, DuMesnil et al. came to similar conclusions. Comparable patterns of ischemic lesions was reported by Brandt et al. in 51 patients $[27,11]$.

Only $51.1 \%$ of all patients received thrombolytic therapy. Except for one patient, who was treated with urokinase, in all other patients rt-PA was used for thrombolytic treatment. 57.8\% showed complete recanalisation and in $37.7 \%$ partial recanalisation was achieved. In only one patient, recanalisation of the basilar artery was not achieved. In contrast, the literature provides varying numbers on basilar artery recanalisation rates: A recanalisation rate of $51 \%$ in 51 patients (urokinase and rtPA) was reported by Brandt et al. [11] and a recanalisation rate of $50 \%$ in 20 patients (urokinase) was reported by DeWitte et al. [28]. The overall range varies from 44 to $80 \%$, suggesting variations in study design and heterogeneity in time to referral as well as high variability of clinical symptoms, as seen also in the present study. Consequently, unless contraindications prevail, immediate thrombolytic treatment should be initiated in order to maintain high rates of recanalisation.

After basilar artery thrombosis, bleeding complications occurred in $12.5 \%$ of the patients, $72.7 \%$ out of them had been treated with rt-PA, while $27.3 \%$ experienced hemorrhage during anticoagulation treatment with heparin. The bleeding rates were $14.5 \%$ in all patients with thrombolytic therapy [11], with similar rates in other reports $9 \%$ [2], 7\% [14], and 10\% [19]. In contrast, Cross et al. [28] identified a rate of symptomatic bleedings after thrombolytic treatment for basilar artery 
thrombosis with rt-PA in $75 \%$. To our knowledge, there are no more comparable data in the literature on re-bleeding in the posterior circulation. ECASS-II had a rate of bleeding complications after thrombolysis for stroke of $35.7 \%$, with rather generous inclusion practice, but these data refer to the anterior circulation only [29]. The most important risk factors for hemorrhage following thrombolytic treatment include increased blood pressure, anti-coagulant or anti-aggregation premedication, increasing latency from symptom onset, old age and leukencephalopathy [29]. Complications seen in the patients included pneumonia, unstable circulation, and bladder infections, all of which can be attributed to immobilization and hospitalization.

Out of all patients suffering from basilar artery thrombosis, 21 patients had a satisfactory neurological outcome, with a mRS ranging from 0 to 2 (the majority of this patients were treated with thrombolytic treatment: (15/21) $71.4 \%$ vs patients without thrombolytic therapy: (6/21) 28.6\%). On the other hand, there was a high number of patients with poor clinical outcome after thrombolytic treatment with a mRS of $5((9 / 12) 75 \%$ with $v s$ (3/12) $25 \%$ without a thrombolysis). Possibly, patients who otherwise would have died from basilar artery stroke will suffer from severe physical impairments after surviving thrombolytic treatment. Based on the mRS, Arnold et al. found a good outcome in $35 \%$ (mRS $0-2)$, poor outcome in $23 \%$ (mRS $3-$ 5 ), and a lethal outcome in $42 \%$ in 40 patients who had been treated with local thrombolytic treatment [19]. We found a lower proportion of patients with fatal outcome $(26.7 \%)$, but the number of patients with severe impairment was higher $(40 \%$.) The proportion of patients with good outcome was similar (33.3\%). The reason for this may be the rate of successful vessel recanalisation. According to Arnold et al. $20 \%$ of all patients had complete and $60 \%$ had partial recanalization [19]. With the other $20 \%$, the basilar artery remained completely blocked. In our study, complete vessel recanalization was achieved in $57.8 \%$, and partial recanalization in $37.7 \%$. The basilar artery remained occluded in only $4.4 \%$ of all cases which were treated with thrombolytic therapy. We conclude that successful thrombolytic treatment leads to a decrease in mortality, and an increase of patients surviving with handicaps. In another study, the percentage of patients who died in spite of successful recanalization after thrombolytic treatment (urokinase or rt-PA) was $35 \%$, while $75 \%$ of the patients died after failure to achieve recanalization [18]. $86 \%$ of the survivors underwent rehabilitation, and $46.3 \%$ of these were discharged to their homes. In our study, the mRS was identified retrospectively according to patients' files. A retrospective survey may lead to variations in the results as compared to direct survey [30]. The mRS represents a subjective scale of physical handicaps and does not include further aspects such as specific activities and everyday life.

Even minor damage to the brainstem may have lethal consequences. Therefore, prognostic factors are difficult to identify. Indicators for putative satisfactory outcome include short duration of basilar occlusion, good collateral circulation, young patient age $[11,39]$, thrombus location (distal better than proximal) [28], and small areas of infarction indicated by minor DWI (diffusion weighed imaging) lesions in MRI [27]. From our patient cohort, we did not succeed in establishing a reliable prognostic score from admission MRI data, owing to varying number, distribution and extent of ischemic lesions, and highly varying extent of clinical impairment that did not correlate with patterns of ischemic lesions.

Consistent with others, our findings suggest that patients with unambiguous basilar artery thrombosis should receive immediate interventional therapy. Therefore, early referral to a hospital center is essential [37]. Tele-neurological communication can help to identify potential candidates for invasive procedures including intra-arterial thrombolytic therapy or mechanical thrombus retrieval at an early stage [26, $31,34]$. In order to achieve good recanalization, a thrombus should be dissolved or removed as early as possible to avoid intolerable duration of ischemia leading to irreversible structural brain damage. There is, however, a certain risk for hemorrhage associated with interventional therapies (approximately $10 \%$ in our study), and the patients and their families should be informed accordingly. ECASS III has demonstrated that systemic thrombolytic treatment can succeed up to 4.5 hours in hemispheric stroke [32]. Extending the time frame for safe thrombolytic treatment to 4.5 hours symptom onset, however, must not end in postponing patients' therapy. Meticulous time management with the best possible order of events is mandatory [37].

It is still a matter of debate which patients should receive interventional therapy, since reliable prognostic parameters are still lacking. Retrospective studies on basilar artery thrombosis and its optimal and differential treatment can provide valuable suggestions and general trends. Major prospective, randomized, multi-center studies would be desirable. Data gained from such an approach would provide relevant information on which patients with a basilar artery thrombosis will likely benefit the most from which type of therapy, and help identify patients who may not need intervention for obtaining a good clinical result [10]. The most informative survey on sequential combination of intravenous and intraarterial thrombolytic treatment after a stroke, the IMS-I Study, included 80 cases from 17 hospitals. In spite of initially severe stroke symptoms with an NIHSS median of 18 before thrombolytic therapy, a high re-opening rate of $80 \%$ was reported. After another three months, $30 \%$ of the patients had a mRS of 0 to 1 , which is a satisfactory clinical result, while only $16 \%$ had died [33]. In addition, the study also focused on patients with thrombosis in the vertebro-basilar territory, but no interpretation of the relation between the respective thrombus localization, recanalization rates, and clinical results was reported.

In conclusion, recent research has suggested that for patients with unambiguous basilar artery thrombosis, the only promising treatment option is early thrombolytic treatment. The major clinical goal is to enable patients to live an independent life after the acute phase and, if required, rehabilitation. Consequently, this challenges the concept of a "thrombolysis at all costs" approach, especially in case of multimorbidity, unidentifiable time frames, and comatose patients with regards to possible poor functional prognosis. Adequate therapy in the sense of an adaptable scheme, starting with immediate maximum therapy following diagnosis ("rescue therapy"), and with defined therapeutic limitations at later stages of the disease course after ensuring poor prognosis may require further testing, depending on clinical conditions and the patients documented or presumable wishes. Some important help in this respect can be provided by consultations of local ethics committees where not 
strictly medical, but also personal, ethical, and religious aspects may be discussed.

\section{REFERENCES}

[1] Labauge R, Pages M, Marty-Double C, Blard JM, Boukobza M, Salvaing P. Occlusion du tronc basilaire. Rev Neurol (Paris) 1981; 137: 545-71.

[2] Hacke W, Zeumer H, Ferbert A, et al. Intra-arterial thrombolytic therapy improves outcome in patients with acute vertebrobasilar occlusion disease. Stroke 1988; 19: 1216-22.

[3] Ferbert A, Brückmann H, Drummen R. Clinical features of proven basilar artery occlusion. Stroke 1990; 21: 1135-42.

[4] Matsumoto K, Satoh K. Topical intraarterial urokinase infusion for acute stroke. In: Hacke W, del Zoppo GJ, Hirschberg M, eds. Thrombolytic Therapy for Acute Ischemic Stroke. New York, NY: Springer Publishing Co, Inc. 1991: 207-12.

[5] Möbius E, Berg-Dammer E, Kühne D, Nahser HC. Local thrombolytic therapy in acute basilar artery occlusion: experience with 18 patients. In: Hacke W, del Zoppo GJ, Hirschberg M eds. Thrombolytic Therapy for Acute Ischemic Stroke. New York, NY: Springer Publishing Co, Inc. 1991: 213-5.

[6] Zeumer H, Freitag HJ, Grzyska U, Neunzig HP. Local intraarterial fibrinolysis in acute vertebrobasilar occlusion. Neuroradiology. 1989; 31: 336-40.

[7] Smith WS. Intra-arterial thrombolytic therapy for acute basilar occlusion: Pro. Stroke 2007; 38: 701-3.

[8] Zeumer H, Hacke W, Kolmann HL, Poeck K. Lokale Fibrinolysetherapie bei Basilaris-Thrombose. Dtsch Med Wochenschr 1982; 107: 728-31.

[9] Mayer TE, Hamann GF, Brueckmann HJ. Treatment of basilar artery embolism with a mechanical extraction device: necessity of flow reversal. Stroke 2002; 33: 2232-5.

[10] Hachinski V. Intra-Arterial Thrombolysis for Basilar Artery Thrombosis and Stenting for Asymptomatic Carotid Disease. Stroke.2007; 38: 721.

[11] Brandt T, von Kummer R, Muller-Kuppers M et al. - Thrombolytic therapy of acute basilar artery occlusion. Variables affecting recanalisation and outcome. Stroke 1996; 27: 875-81.

[12] Moufarrij NA, Little JR, Furlan AJ, Leatherman JR, Williams GW. Basilar and distal vertebral artery stenosis:long-term follow-up. Stroke 1986;17: 938-42.

[13] The Warfarin-Aspirin Symptomatic Intracranial Disease (WASID) Study Group. Prognosis of patients with symptomatic vertebral or basilar artery stenosis. Stroke 1998; 29: 1389-92.

[14] Zeumer H, Freitag HJ, Zanella F, Thie A, Arning C. Local intra-arterial fibrinolytic therapy in patients with stroke: urokinase versus recombinant tissue plasminogen activator (r-TPA). Neuroradiology 1993; 35: 159-62.

[15] Möbius E, Berg-Dammer E, Kühne D, Kunitsch G, Nahser HC. Lokale intraarterielle Fibrinolyse bei A. basilaris-Verschluß mit progredientem Hirnstamminfarkt. Akt Neurol 1989; 16: 184-90.

[16] Bockenheimer S, Reinhuber F, Mohs C. Intraarterielle Thrombolyse hirnversorgender Gefäße. Radiloge 1991; 31:210-5.

[17] Wijdicks EFM, Scott JP. Outcome in patients with acute basilar artery occlusion requiring mechanical ventilation. Stroke 1996; 27: 1301-3.

[18] Sliwka U, Mull M, Stelzer A, Diehl R, Noth J. Long-term follow-up of patients after intraarterial thrombolytic therapy of acute vertebrobasilar artery occlusion. Cerebrovasc Dis 2001; 12 (3): 214-9.

[19] Arnold M, Nedeltchev K, Schroth G et al. Clinical and radiological predictors of recanalisation and outcome of 40 patients with acute basilar artery occlusion treated with intra-arterial thrombolysis. J Neurol Neurosurg Psychiatry 2004; 75: 857-62.
[20] Deutsche Diabetes-Union. Deutscher Gesundheitsbericht Diabetes 2008. Vorgelegt von der deutschen Diabetes-Union und dem nationaöen Aktionsforum Diabetes mellitus; www.diabetes-union.de.

[21] Renz-Polster H, Braun J. Basislehrbuch Innere Medizin. Urban \& Fischer 2004.

[22] Hoffmann E, Gerth A, Janko S, Hahnefeld A, Dorwarth U, Remp T, Steinbeck G. Herzrhythmusstörungen: geschlechtsspezifische Unterschiede. Intensivmedizin und Notfallmedizin. Steinkopff 2003; vol. 40(8)..

[23] Schunkert H. Prähypertonie - eine Grenzwertbestimmung. Dtsch Med. Wochenschrift 2006; 131: H17-9.

[24] Hense HW. Epidemiologie der arteriellen Hypertonie und Implikationen für die Prävention. Dtsch Med. Wochenschrift 2000; 125: $1397-402$.

[25] Diener HC, Putzki N, Berlit P, et al. Leitlinien für Diagnostik und Therapie in der Neurologie; vierte überarbeitete. Auflage. Georg Thieme Verlag 2008; S233-S87.

[26] Müller R, Pfefferkorn T, Vatankhan B, et al. Admission facility is associated with outcome of basilar artery occlusion. Stroke 2007; 38: 1380-3.

[27] DuMesnil R, Neumann-Haefelin T, Berkefeld J, Sitzer M, Lanfermann H. Magnetic resonance imaging in basilar artery occlusion. Arch Neurol 2002; 59: 398-402.

[28] Cross deWitte T, Derdeyn CP, Moran CJ. Bleeding complications after basilar artery fibrinolysis with tissue plasminogen activator. Am J Neuroradiol 2001; 22: 521-3.

[29] Larrue V, Von Kummer R, Müller A, Bluhmki E. Risk factors for severe hemorrhagic transformation in ischemic stroke patients treated with recombinant tissue plasminogen activator (rt-PA). A secondary analysis of the European-Australasian acute stroke study (ECASS II). Stroke 2001; 32: 438-41.

[30] Quinn TJ, Ray G, Atula S, Walters MR, Dawson J, Lees KR. Deriving modified Rankin scores from medical case-records. Stroke 2008; 39: 3421-3.

[31] Ickenstein GW, Isenmann S, Fiehler J, Gahn G, Schumacher M, Klisch J. Thrombolysis in Neuromedicine - Bridging concepts for the management of acute brain infarction with occlusion of intracranial vessels. Clin Neuroradiol 2008; 18: 88-97.

[32] Hacke $\mathrm{W}$ et al. Leitlinien für Diagnostik und Therapie in der Neurologie. Georg Thieme Verlag Stuttgart 2008 (4. überarbeitete Auflage); $645 \mathrm{ff}$.

[33] Broderick J. IMS Study Investigators. Combined intravenous and intraarterial recanalisation for acute ischemic stroke: the interventional management of stroke study. Stroke 2004; 35: 904-11.

[34] Audebert HJ, Kukla C, Claranau SC, et al. Telemedicine for safe and extended use of thrombolysis in stroke: the telemedic pilot project for integrative stroke care (TEMPiS) in Bavaria. Stroke 2005; 36: 287-291.

[35] Candelise L, Gattinoni M, Bersano A, Micieli G, Sterzi R, Morabito A PROSIT Study Group. Stroke-unit care for acute stroke patients: an observation follow-up study. Lancet 2007; 369: 299.

[36] Chalela JA, Kidwell CS, Nentwich LM, et al. - Magnetic resonance imaging and computed tomography in emergency assessment of patients with suspected acute stroke: a prospective comparison. Lancet 2007; 369 No. $9558: 293-8$

[37] Kamper L, Rybacke K, Mansour M, Winkler SB, Kempkes U, Haage $\mathrm{P}$. Time management in acute vertebrobasilar occlusion. Cardiovasc Intervent Radiol 2009; 32: 226-32.

[38] Pfefferkorn T, Mayer TE, Opherk C, et al. Staged escalation therapy in acute basilar artery occlusion: intravenous thrombolysis and on-demand consecutive endovascular mechanical thrombectomy: preliminary experience in 16 patients. Stroke 2008; 39: 1496-500.

[39] Preul C, Joachimski F, Witte OW, Isenmann S. Bilateral vertebral artery dissection after chiropractic maneuver. Clin Neuroradiol 2010; 20(4): 255-9. 